\title{
A New Robotic System for Visually Controlled Percutaneous Interventions under CT Fluoroscopy
}

\author{
Michael H. Loser ${ }^{1}$, Nassir Navab ${ }^{2}$ \\ ${ }^{1}$ Siemens AG, Medical Engineering, Henkestraße 127, D-91052 Erlangen, Germany \\ and Institute for Microsystem-Technology, University of Freiburg, Germany \\ michael.loser@med.siemens.de \\ ${ }^{2}$ Siemens Corporate Research, 755 College Road East, Princeton, NJ 08540, USA \\ navab@scr.siemens.com
}

\begin{abstract}
Minimally invasive CT-guided interventions are an attractive option for diagnostic biopsy and localized therapy delivery. This paper describes the concept of a new prototypical robotic tool, developed in a preliminary study for radiological image-guided interventions. Its very compact and light design is optimized for usage inside a CT-gantry, where a bulky robot is inappropriate, especially together with a stout patient and long stiff instruments like biopsy needles or a trocar. Additionally, a new automatic image-guided control based on "visual servoing" is presented for automatic and uncalibrated needle placement under CT-fluoroscopy. Visual servoing is well established in the field of industrial robotics, when using CCD cameras. We adapted this approach and optimized it for CT-fluoroscopy-guided interventions. It is a simple and accurate method which requires no prior calibration or registration. Therefore, no additional sensors (infrared, laser, ultrasound, etc), no stereotactic frame and no additional calibration phantom is needed. Our technique provides accurate $3 \mathrm{D}$ alignment of the needle with respect to an anatomic target. A first evaluation of the robot using CT fluoroscopy showed an accuracy in needle placement of \pm 0.4 $\mathrm{mm}$ (principle accuracy) and $\pm 1.6 \mathrm{~mm}$ in a small pig study. These first promising results present our method as a possible alternative to other needle placement techniques requiring cumbersome and time consuming calibration procedures.
\end{abstract}

\section{Introduction}

CT-guided percutaneous biopsy, drainage, and tumor ablation are widely accepted and often performed, since CT provides reproducible high-resolution images with good differentiation of soft tissue. Efficient and safe CT-guided percutaneous interventions require accurate needle placement, particularly for small or deeply situated target areas. To facilitate CT-guided interventions and to support the physician in conducting needle placement outside the CT-gantry, different guiding systems have been developed. Handheld guidance devices, consisting of a needle holder with an attached protractor and a simple level indicator, provide simple but useful support [1]. Another supporting approach for manually guided needle placement outside the CT-gantry is the use of laser guidance system [2][3][4]. Although stereotactic systems in combination with preoperative CT-imaging are commonly used in neurosurgery for localizing brain lesions, experience in other parts of the body is relatively limited [5]. Many

S.L. Delp, A.M. DiGioia, and B. Jaramaz (Eds.): MICCAI 2000, LNCS 1935, pp. 887-896, 2000.

(C) Springer-Verlag Berlin Heidelberg 2000 
techniques have been developed which integrate robotic guidance of end effectors with image based stereotactic procedures using a variety of registration techniques. First experiments on robot guided interventions combined with CT imaging have been performed by Kwoh et al. in 1985 [6]. The target applications in these early trials in using a robot for stereotactic interventions with a CT scanner were stereotactic brain surgery in combination with a head frame [7][8][9]. Glauser et al. also used a stereotactic head frame to register the robot and image space, but are able to perform needle placement under active CT surveillance to confirm the position of their end effector [10]. Similarly, Masamune et al. performed needle placement within the CT scanner and registered using a stereotactic head frame [11]. A new approach was developed by Susil et al. [12], who used a special localization frame fixed to a robot's end effector to guide a needle inside a CT gantry. Using the cross section of this frame in the image, they can compute the pose of the end effector holding the needle relative of the CT image. Disadvantage of this method is the need of additional space for moving this frame inside the gantry.

Our robotic prototype follows a new approach, providing a very small and compact intelligent tool together with direct $\mathrm{CT}$ surveillance for automatic needle alignment. We tried to imitate inside the CT-gantry what the physician is doing while manipulating the needle manually. He is visually guiding the needle - visible in the CT-image until it is aligned with the target structure. To perform this intervention manually has basic disadvantages. Firstly, the radiation exposure for the physician especially for his hands. Because of this, he needs a special needle holder in order not to place his hands in the X-ray beam. Secondly, the manual manipulation of the needle inside the CTgantry often forces the physician to an inconvenient posture during the intervention. With the development of our prototype, guiding the needle automatically using principles of visual servoing, we avoid these disadvantages and demonstrate a simple and intelligent tool for precise and fast needle placement under CT-fluoroscopy.

\section{The Robot's Design}

The goal was to develop an optimized needle-guiding system for automatic or remote controlled percutaneous needle alignment inside a CT gantry. The system should help to minimize radiation exposure and inconvenient posture for the physician manipulating the needle manually inside the CT gantry. Especially complicated procedures are expected to be performed with improved safety, accuracy and speed.

The resulting concept is a prototypical surgical robotic system shown in figure 1. Its very small and compact design (length $365 \mathrm{~mm}$, weight $590 \mathrm{~g}$ ) with a radiolucent distal part is optimized for precise needle manipulation inside a CT-gantry. This robot could be controlled via joystick or - as described in the next section - automatically using visual servoing, an image based control approach. Best performance will be achieved by using CT fluoroscopy which allows real-time imaging and is increasingly used in interventional procedures [13][14][15]. Our robotic tool consists of a very compact parallel-gear that allows needle rotation around two perpendicular axes. These two rotational degrees of freedom allow us to rotate the needle in $3 \mathrm{D}$ around a certain rotation point which is defined by the geometry of the design. Two servo 


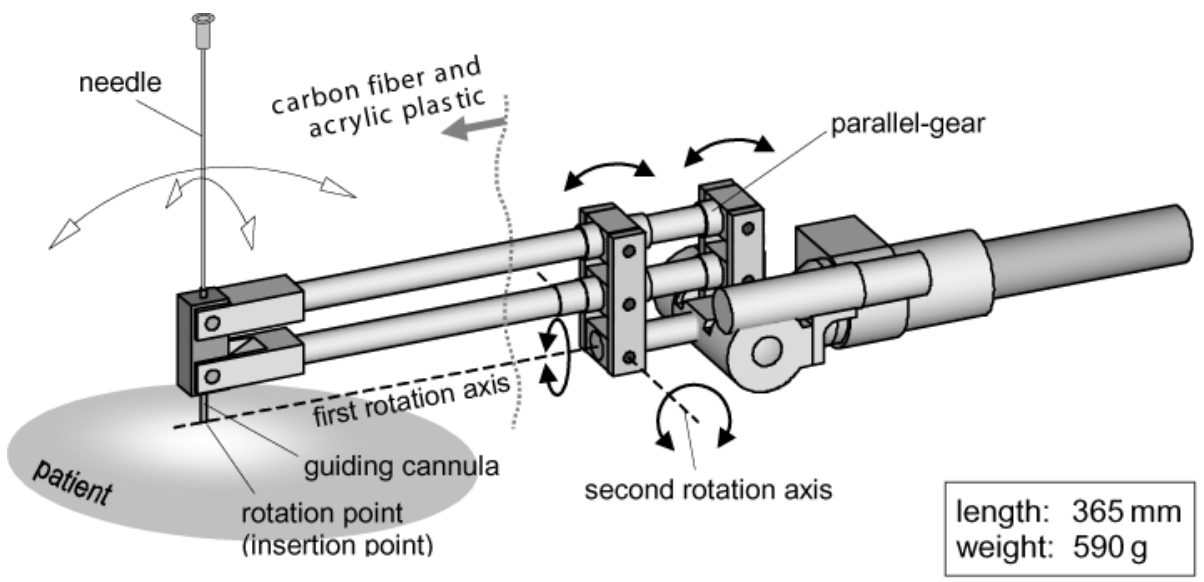

Fig. 1 The prototype has two perpendicular rotational axis that allow 3D needle-rotation around a fixed rotation point. The parallel-gear allows a very compact design for the use inside a CT-gantry. The whole system is very small and lightweight designed which provides enough space between robot and the patient, even for strongly tilted needles. All cables are integrated inside the construction.

drives together with precise micro-encoders are responsible for accurate needle motion. The resolution of needle rotation around the two axis is $\pm 0.04^{\circ}$. This parallel design allows the easy separation between a radiolucent distal part, where only carbon fiber and acrylic plastic is used (except the metallic guiding cannula), and a very rigid metallic construction containing all servo drives, encoders and limit switches. Apart from CT-guided interventions, this radiolucent distal part of the robot makes X-ray imaging (fluoroscopy) possible, where a guiding tool should not obscure patient's anatomy in the X-ray image. Additionally a special radiolucent needle transmission is integrated at the guiding cannula and allows the remote controlled needle insertion. All electric cables for motors, encoders and limit switches are integrated inside the construction. The robot can be attached to a passive arm while the electric contact to the robot is obtained by a special electric plug connection. The passive arm is a sevendegree of freedom manipulator that may be locked in the desired position. The robotic tool is a unique miniaturized radiolucent construction which provides motorized or manual needle insertion. The needle injection is powered by a small DC motor integrated in the parallel-structure of the construction. A special feature of the prototype's needle drive is that it grasps the distal end of the needle, not the needle head. This significantly reduces the unsupported length of the needle, thus minimizing the lateral flexure during injection and increasing the accuracy.

\section{Visual Servoing - An Image Based Control for the Robot}

Most robot applications require interaction of the robot end-effector with objects in the work environment. Conventional robot control focuses on positioning the endeffector accurately in a fixed world coordinate frame. To achieve precise and reliable interaction with targets in the workspace, these objects have to be consistently and ac- 
curately located in the same world coordinate system. Uncertainties in the end-effector position or the object position will lead to a position mismatch and potentially to the failure of the operation. The problem can be minimized by using precise joint angle encoders. Another solution, which has received attention in the manufacturing field, is the visual control of manipulators based on cameras. It offers substantial advantages, when working with targets placed at unknown locations or with flexible or inaccurate manipulators. This can support accurate free-space motion in unstructured environments, which can evolve over time. An excellent overview of the visual servo control of robot manipulators and applications, is given by Corke [13].

Traditionally, visual sensing and manipulation are combined in an open-loopfashion, 'looking-then-moving'. The accuracy of the operation depends directly on the accuracy of the visual sensor and of the manipulator. A more accurate alternative is to 'close' the loop by a visual-feedback control loop. If the robot system incorporates vision based on task level programming using joint feedback information, it is called a dynamic look-and-move control. If servoing is done directly on the basis of image features without joint feedback, it is called image based servoing as proposed by Weiss [17]. The visual control approach that we implemented for our robotic tool using CT fluoroscopy is a dynamic look-and-move control and is described in section 3.2 .

\subsection{Visual Servoing in Medicine}

A central concern of visual servoing research for industrial robotics are dynamic issues of the visual control, since the economic justification is frequently based upon cycle time. High sample rate for the vision sensor and robot control, and low latency and high-bandwidth communications are critical to a short settling time [18]. However, in medicine, the situation is radically different. In X-ray medical imaging the imaging sample rate and dose are first determined from diagnostic or clinical considerations. While in industrial robot applications a high imaging sample rate would be desirable because of dynamic issues, in X-ray imaging this could result in high radiation exposure for both patient and physician. Furthermore, guidelines for industrial robots suggest that robots should not be powered when people are in the vicinity, whereas this would be inappropriate in the OR, where robots are working very closely together with surgeons and the patient [19]. Safety aspects are the main reason why medical robots are generally moving very slowly. Thus, sophisticated dynamic visual control issues are of lower importance in medicine.

Since the use of medical robots has only begun in the last decade, there are currently not many visual servoing systems developed in the medical field. Most of these systems using visual control have been proposed in the field of minimally invasive surgery to control motion of a camera or an endoscopic instrument [20][21][22]. One recent development is a laparoscopic guiding system for an AESOP 1000 robot (Computer Motion, Goleta, CA, USA), developed by the German Aerospace Research Establishment (DLR) [23]. Using color image segmentation this system locates the tip of a laparoscope provided with a green mark. This image feature guides the laparoscopic camera so that the instrument's tip is always at a certain position in the image. Salcudean et al. [24] proposes a special counterbalanced robot for positioning an ul- 
trasound probe. He uses visual servoing for robot image feature tracking in the ultrasonic image plane. A new approach for semi-automatic needle placement under X-ray fluoroscopy using visual servoing is described by Loser et al. [25]. To the authors knowledge, this paper presents the first experimental results on visual servoing using CT fluoroscopy.

\subsection{Visual Servoing for CT Fluoroscopy}

In this section we describe the principle of our approach for automatic and uncalibrated needle placement with CT-fluoroscopy-guidance for two different scenarios:

(a) The target structure and the needle insertion point are located in the same CTimage plane. Therefore, the access trajectory for the needle and thus the complete insertion procedure is visible in one image plane.

(b) The target and the insertion point are located in two different CT-image planes. In case that target and insertion point could not be located in the same image plane by tilting the CT gantry, the access trajectory and thus the insertion procedure is not directly visible in the CT-image.

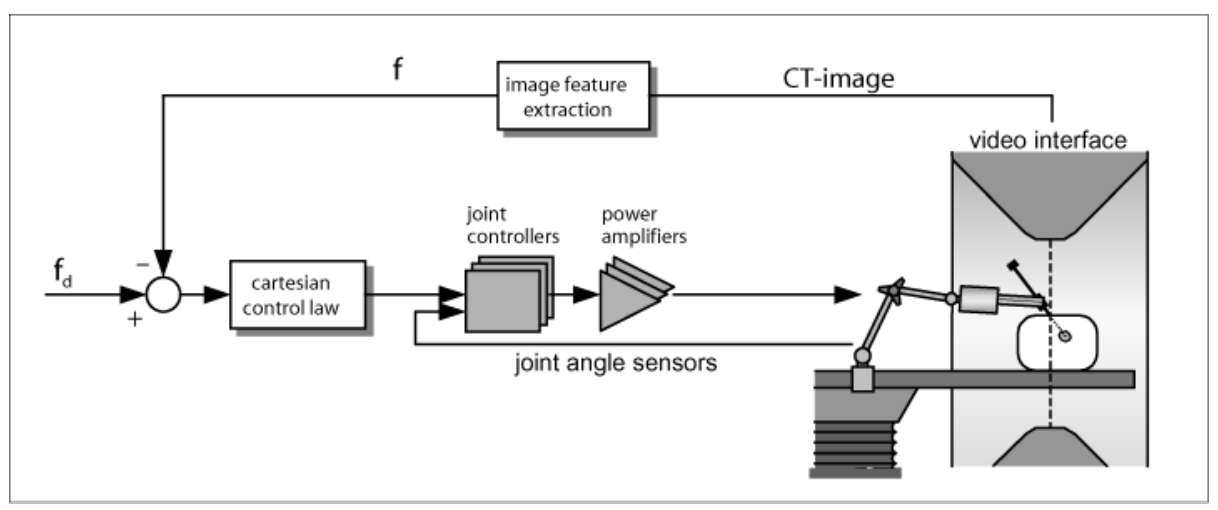

Fig. 2 Controller block diagram of visual servoing feedback loop for automatic needle alignment using CT fluoroscopy guidance.

The controller block diagram of our CT-guided visual servoing feedback loop is shown in figure 2. Using an image based visual control the elements of the task are specified in image feature space, not in world space. Mostly the real world task is described by one or more image space tasks [26]. For instance, the robot motion is controlled to achieve desired conditions on the image, e. g. the alignment of a needle with a target point in the scene. This means, the location of features on the image plane are directly used for feedback. In our application there are two image features to control:

1) The first image feature is the visible needle length $\lambda$ in the image, which is needed to control the automatic tilting of the needle into the image plane. 
2) The second image feature is the deviation angle $\Delta \phi$ between the actual and the desired needle orientation in the image. This parameter is used for the needle alignment feedback loop in the image plane.

\section{(a) Scenario 1: Insertion of needle in image plane}

First the robot has to be placed manually at the desired insertion point on the patients skin. The tip of the guiding cannula (rotation point) is now identical with the insertion point. In the next step the CT-table is moved automatically while imaging, until the tip of the guiding cannula is visible in the image (needle might be already put in the guide). Using image processing, this cannula is automatically detected in the image (see fig. 3a). Now the robot tilts the guiding cannula as long as it appears with maximum length in the CT-image. But the robotic system still does not know the orientation of the CT-image plane in the robot's coordinate frame. Therefore, we need a second needle position in the CT-image plane in order to define its position and orientation in robot's coordinate frame. So the robot rotates the needle around its first rotation axis (see fig. 1) with a certain amount and tilts the needle again into the image plane until its visible with maximum length. Now the CT-image plane, containing these two needle positions, is defined in the robot's coordinate frame (see fig. 3b). This registration between robot and the CT-image plane is part of the automatic alignment procedure. The next step is to rotate the needle in this image plane until alignment with the target is achieved. This is done by rotating the needle as long in the image plane as the measured deviation angle in the image vanishes (see fig. 3c). This whole procedure is done automatically while the needle insertion itself can be performed manually or with remote control by the physician.

\section{(b) Scenario 2: insertion of needle tilted to image plane}

In this second scenario the target structure and the insertion point are not laying in the same CT-image plane. Therefore, the CT-image containing the target structure has to be identified, then the physician chooses the target point in this image (image coordinates $\left.x_{1}, y_{1}\right)$. After defining the best access trajectory the robot's guiding cannula is placed at the resulting insertion point on the patient's skin (needle might be already put in the guide). The physician moves the CT-table until the needle tip (insertion point) becomes visible in the image (see fig. 4a). Now the image coordinates of target $\left(x_{1}, y_{1}\right)$ and insertion-point $\left(x_{2}, y_{2}\right)$ are defined. Since we know the CT-table translation $\Delta \mathrm{T}$ between the target image plane and the insertion-point image plane, the complete three-dimensional settings are known in CT-image space. In the next step the robot has to be registered to the CT-image plane, as described in scenario 1 (see fig. $4 \mathrm{~b}$ ). After the image plane is known in the robot's coordinate frame the prototype can adjust the needle orientation which aligns the needle with the target. In this second scenario, needle insertion must be performed remotely by the physician. While in scenario 1 the needle insertion and deviations can be directly observed in the image, this is not possible in this second scenario. But during needle insertion the system ensures by moving the CT-table while imaging, that the needle tip is always visible as a bright dot in the CT-image. Also projected into the image is the computed desired insertion trajectory (fig. 4c), so that the physician can observe on the monitor - even in this second scenario - the deviation between desired and actual needle tip position during remote controlled insertion. 


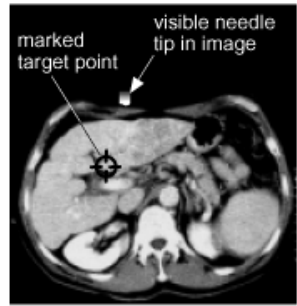

(a)

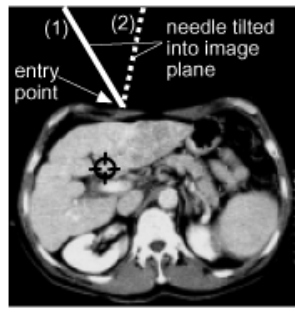

(b)

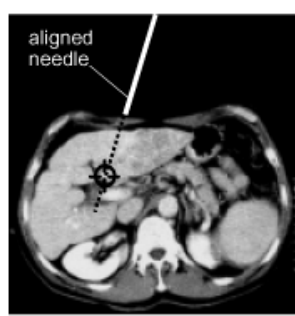

(c)

Fig. 3

The automatic needle alignment process in case that insertion point and target are located in the same CT-image plane (scenario 1).

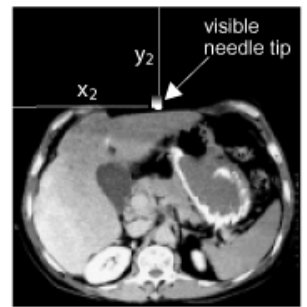

(a)

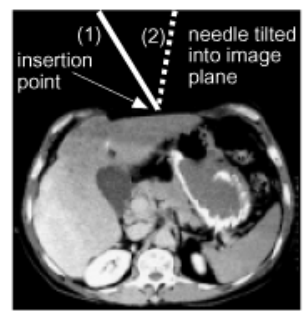

(b)

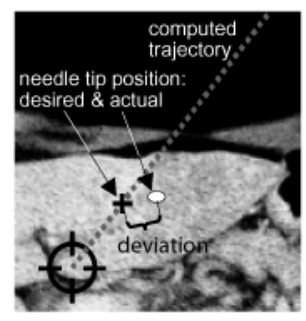

(c)

Fig. 4

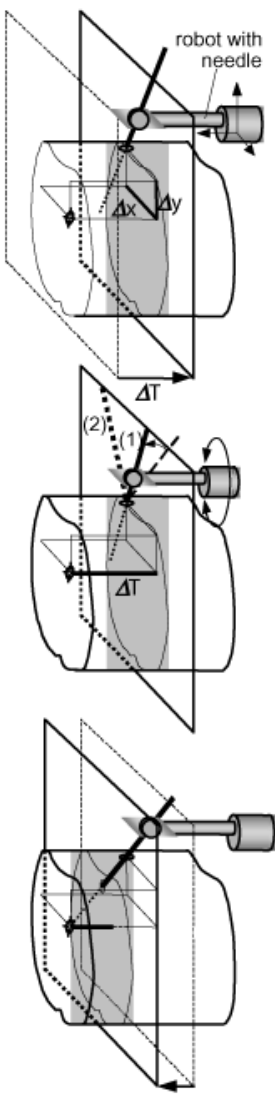

The automatic needle alignment process in case that insertion point and target are located in two different CT-image planes (scenario 2).

\section{Setup of Robotic Prototype together with a CT Scanner}

We conducted our first experiments in automatic needle placement with a Somatom Plus4 CT scanner (Siemens AG, Forchheim, Germany) with CT-fluoroscopy (CARE vision) at our CT laboratory. Figure 5 shows the experimental setup. The prototype with attached needle is fixed to a passive arm. For these first experiments we used a "CT-table simulator" to perform translational motion. This simulator consists of a linear drive on which the passive arm and an acrylic plate as seat for test objects are fixed. In preliminary tests a small metal ball with a diameter of $2 \mathrm{~mm}$ is used as target. In a second test series we performed image guided needle placement with different pig organs (kidney, liver, lung) as phantom (see fig. 6). We used the shell of a water melon to simulate the abdominal wall (see fig. 6 and 7) and to stabilize the underlying structures. 


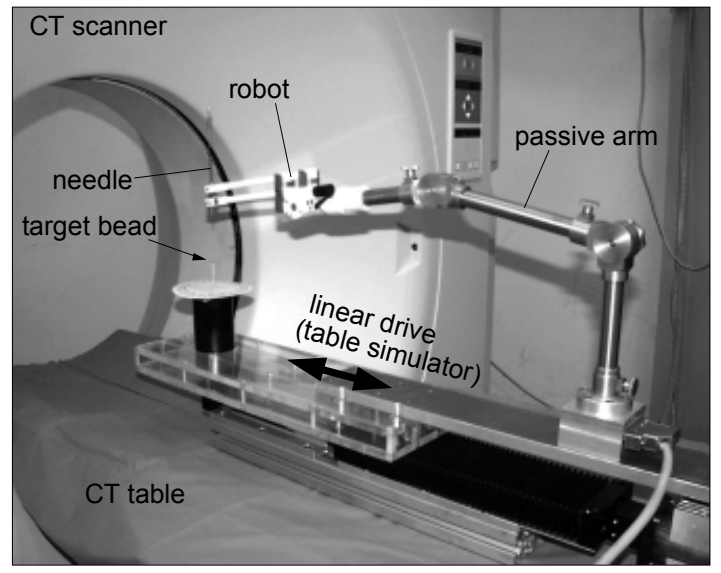

Fig. 5 CT-setup. The robot is fixed to a passive arm. We used a linear drive as "table simulator" to perform translational motion. In preliminary experiments a small metal ball is used as target (without phantom).

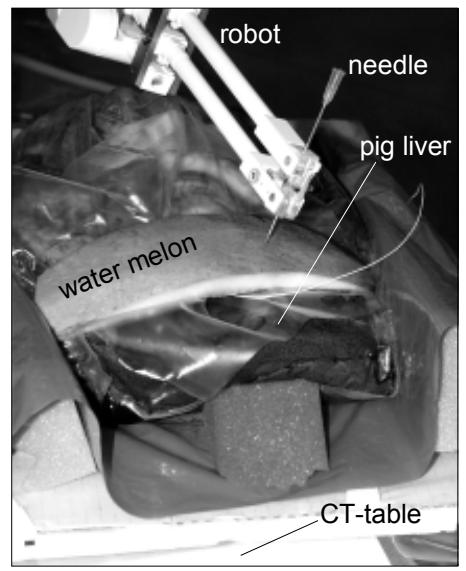

Fig. 6 In a second test series different pig organs are used for needle placement. Here liver puncture is performed inside the gantry.

\section{Evaluation of Automatic CT-Fluoroscopy Guided Needle Placement}

In preliminary experiments the phantom consisted only of a $2 \mathrm{~mm}$ metal ball without any additional structures between rotation point and target (fig. 5). The purpose of these tests was the evaluation of principle accuracy of our automatic alignment technique described in section 3.2, without the influence of needle bending and drift during insertion. The distance between needle tip and target location was approximately $70 \mathrm{~mm}$. To determine the remaining 3D deviation vector $\Delta$ between the needle axis and the target midpoint after needle alignment, we took digital images with a CCDcamera from two known viewing directions. After analyzing these two CCD-images we could precisely compute the desired deviation vector $\Delta$ for all alignment trials. The mean deviation in these preliminary experiments was about 0.4 millimeters. The authors want to emphasize that this is a kind of technical accuracy of the applied method.

In order to obtain results that are more clinically relevant, we performed several test series using different pig organs as phantom (see fig. 7). After automatic alignment, incremental manual advancement of the needle was performed, followed by intermittent CT-fluoroscopy to assess the needle position after each increment. Results are shown in table 1 . We achieved a mean deviation over all series with pig organs of $1.6 \mathrm{~mm}$. In these laboratory experiments we performed CT-fluoroscopy scanning continuously during the alignment process. For clinical usage together with patients we propose intermittent CT-fluoroscopy for the visual control of the needle. In conjunction with further optimization of the control loop, this would result in significantly shorter CT-fluoroscopy scan time and radiation dose. For sterilization of the robot, we propose the use of surgical drape for the whole device except the guiding cannula, which is easily detachable and sterilizable separately. 


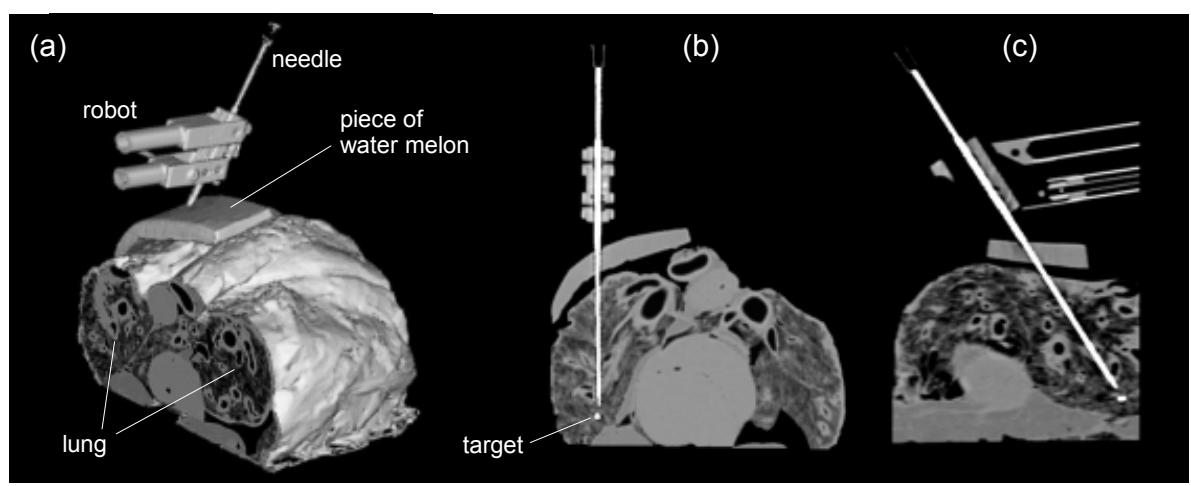

Fig. 7 Needle placement with pig lung. To determine the deviation between needle axis and target after needle insertion, we performed a high resolution spiral scan (a) and measured the deviation out of two perpendicular slices along the needle axis (b and $c)$.

\begin{tabular}{|c|c|c|c|c|c|}
\hline $\begin{array}{c}\text { series } \\
\text { no. }\end{array}$ & $\begin{array}{c}\text { CT fluoros } \\
\text { copy time }\end{array}$ & $\begin{array}{c}\text { insertion } \\
\text { depth }\end{array}$ & location & $\begin{array}{l}\text { needle orienta- } \\
\text { tion to image }\end{array}$ & $\begin{array}{c}\text { mean } \\
\text { deviation }\end{array}$ \\
\hline 1 & $39 \mathrm{sec}$ & $7.2 \mathrm{~cm}$ & kidney & tilted & $1 \mathrm{~mm}$ \\
\hline 2 & $40 \mathrm{sec}$ & $7.9 \mathrm{~cm}$ & liver & in image plane & $2 \mathrm{~mm}$ \\
\hline 3 & $40 \mathrm{sec}$ & $8.7 \mathrm{~cm}$ & liver & tilted & $3 \mathrm{~mm}$ \\
\hline 4 & $38 \mathrm{sec}$ & $5.8 \mathrm{~cm}$ & lung & in image plane & $1 \mathrm{~mm}$ \\
\hline 5 & $40 \mathrm{sec}$ & $7.7 \mathrm{~cm}$ & lung & tilted & $1 \mathrm{~mm}$ \\
\hline
\end{tabular}

Tab. 1

Results of needle placement in different pig organs. We achieved a mean deviation over all series of $1.6 \mathrm{~mm}$. target: metal bead (Ø2 $\mathrm{mm})$

\section{Conclusion}

In this paper we presented a new prototypical robot for image-guided interventions. With a very compact and light design, this robot is optimized for usage inside a CT gantry. Furthermore, we proposed a simple and accurate method for automatic needle placement. Using visual servoing under CT fluoroscopy guidance in two possible scenarios. The only human interaction required by the system is the choice of the needle insertion point on the patient and the manual definition of the target point on the computer display showing the CT-images. In the case of manual needle insertion this last interaction is also performed by the physician. Our method requires no additional sensors (infrared, laser, ultrasound, etc), no stereotactic frame and no prior calibration using a phantom or fiducial markers. The approach has been tested with our prototype in different experiments using pig organs as phantom. Promising first results present this method as a possible alternative to other needle placement techniques, which require cumbersome and time consuming calibration procedures.

\section{References}

1. A. M. Palestrant, "Comprehensive approach to CT-guided procedures with a hand-held guidance device”, Radiology, vol. 174, pp. 270-272, 1990.

2. H. Ishizaka, T. Katsuya, Y. Koyama, H. Ishijima, T. Moteki, et al. "CT-guided percutaneous intervention using a simple laser director device”, AJR, 170(3), pp. 745-746, 1998. 
3. A. Gangi, B. Kastler, J. M. Arhan, A. Klinkert, et al. "A compact laser beam guidance system for interventional CT”, J. of Comp. Assisted Tomography, 18, pp. 326-328, 1994.

4. C. Frahm, W. Kloess, H.-B. Gehl, et al., "First experiments with a new laser-guidance device for MR- and CT-guided punctures", European Radiology, vol. 5, p. 315, 1994.

5. G. Onik, P. Costello, E. Cosman, et al., "CT body stereotaxis: an aid for CT-guided biopsies", AJR, vol. 146, pp. 163-168, 1986.

6. Y. S. Kwoh, I. S. Reed, J. Y. Chen, H. M. Shao, et al., “A new computerized tomographicaided robotic stereotaxis system“, Robotics Age, vol. 7, pp.17-22, 1985.

7. R. F. Young, "Application of robotics to stereotactic neurosurgery", Neurological Research, vol. 9, pp. 123-128, 1987.

8. Y. S. Kwoh, J. Hou, E. A. Jonckeere, S. Hayati, “ A Robot with improved absolute positioning accuracy for CT guided stereotactic brain surgery”, IEEE Transactions on Biomedical Engineering, 35(2), pp. 153-160, 1988.

9. J. M. Darke, M. Joy, A. Goldenberg, et al. "Computer and robotic assisted resection of brain tumors", Proc. 5th Intern. Conference on Advanced Robotics, pp. 888-892., 1991.

10. D. Glauser, H. Frankenhauser, M. Epitaux, J.-L. Hefti, A. Jaccottet, "Neurosurgical robot Minerva, first results and current developments", in Proc. 2nd Symp. On MRCAS , 1995.

11. K. Masamune, et al., "A newly developed stereotactic robot with detachable driver for neurosurgery”, in Proc. MICCAI 1998, pp. 215-222, 1998.

12. R. C. Susil, J. H. Anderson, R. H. Taylor, "A single image registration method for CT guided interventions”, in Proc. MICCAI 1999, pp. 798-808 , 1999.

13. K. Katada, R. Kato, H. Anno, Y. Ogura, S. Koga, Y. Ida, et al., "Guidance with real-time CT fluoroscopy: early clinical experience”, Radiology, 200(3), pp. 851-856, 1996.

14. J. J. Fröhlich, B. Saar, M. Hoppe, et al., "Real-time CT-fluoroscopy for guidance of percutaneous drainage procedures", Journal Vasc. Interv. Radiology, 9(5), pp. 735-740, 1998.

15. B. Daly, P. A. Templeton, “ Real-time CT fluoroscopy: evolution of an interventional tool”, Radiology, 211(2), pp. 309-315, 1999.

16. P. Corke, "Visual control of robot manipulators - a review", in Visual Servoing, Ed. K. Hashimoto, World Scientific, Singapore, vol. 7, pp. 1-31, 1993.

17. L. E. Weiss, A. C. Sanderson, C. P. Neumann, "Dynamic sensor-based control of robots with visual feedback”, IEEE J. Robotics and Automation, vol. RA-3, pp. 404-417, 1987.

18. P. I. Corke, M. C. Good, "Dynamic effects in visual closed-loop systems", IEEE Trans. Robotics and Automation, 5(12), 1996.

19. B. Davis, "Safety of Robots in Surgery", in Proc. of 2nd Workshop on Medical Robotics, IARP , Ed. R. Dillmann et al., p. 101, 1998.

20. R. H. Taylor, J. Funda, B. Eldridge, D. Larose, et al., "A telerobotic assistant for laparoscopic surgery", IEEE Journal Engin. in Medicine and Biology, 14(3), pp. 279-288, 1995.

21. A. Casals, J. Amat, D. Prats, E. Laporte, "Vision guided robotic system for laparoscopic surgery", in Proc. Int. Conf. on Advanced Robots, pp. 33-36, 1995.

22. C. Lee, Y. F. Wang, D. R. Uecker, et al., "Image analysis for automated tracking in robotassisted endoscopic surgery", in Proc. Int. Conf. on Pattern Recognition, pp. 88-92, 1994.

23. G.-Q. Wei, K. Arbter, G. Hirzinger, "Real-time visual servoing for laparoscopic surgery", IEEE Eng. In Medicine and Biology, 16(1), pp. 40-45, 1997.

24. S. E. Salcudean, G. Bell, S. Bachmann, et al. "Robot-assisted diagnostic ultrasound - design and feasibility experiments", in Proc. MICCAI 1999, pp. 1062-1071, 1999.

25. M. H. Loser, N. Navab, B. Bascle, R. H. Taylor, "Visual servoing for automatic and uncalibrated percutaneous procedures", in Proc. SPIE Medical Imaging, pp. 270-281, 2000.

26. D. B. Westmore, W. J. Wilson, "Direct dynamic control of a robot using an end-point mounted camera and Kalman filter position estimation", in Proc. IEEE Int. Conf. on Robotics and Automation, pp. 2376-2384, 1991. 